\title{
Neutron Spin-Echo Study of Dynamics of Hydrophobically Modified Polymer-Doped Surfactant Bilayers
}

\author{
Bing-Shiou Yang, ${ }^{\dagger}$ J yotsana Lal, „ Mihaela Mihailescu, $§$ Michael Monkenbusch, $§$ \\ Dieter Richter, $\$$ J ohn S. Huang, ${ }^{\dagger}$ J oachim Kohn," William B. Russel, ${ }^{*},{ }^{\dagger}$ and \\ Robert K. Prud'homme \\ Department of Chemical Engineering, Princeton University, Princeton, New J ersey 08544, \\ Intense Pulsed Neutron Source, Argonne National Lab, Argonne, Illinois 60439, \\ Institut für Festkörperforschung - Forschungszentrum J ülich, D-52425 J ülich, Germany, and \\ Department of Chemistry, Rutgers University, Piscataway, New J ersey 08854
}

Received May 2, 2001. In Final Form: October 9, 2001

\begin{abstract}
Wecharacterizetheeffect of adsorbed hydrophobically modified polymers (hm-polymers) on thedynamics of surfactant bilayers by small-angle neutron scattering and neutron spin-echo spectroscopy. Two kinds of hm-polymers, (a) hydrophobically modified poly(acrylate) with tetradecyl $\left(\mathrm{C}_{14}\right)$ sidegroups randomly grafted to the poly(acrylate) backbone and (b) poly(PEG $6 \mathrm{k}^{-l y s i n e-s t e a r y l a m i d e) ~ w i t h ~ e q u a l l y ~ s p a c e d ~}$ hydrophobes, are added to bilayers of penta(ethylene glycol) dodecyl ether $\left(\mathrm{C}_{12} \mathrm{E}_{5}\right)$ and hexanol. Both bare and polymer-doped membranes exhibit a stretched exponential relaxation in the form of $S(\vec{q}, t) \cong S(\vec{q})$ $\exp \left[-\left(\Gamma_{\mathrm{q}} \mathrm{t}\right)^{2 / 3}\right]$, where $\mathrm{S}(\mathrm{q})$ is the static structure factor and $\Gamma_{\mathrm{q}}$ is the relaxation rate. The relaxation rate depends subtly on the surface coverage of polymer. At high surface coverage, polymer slows down the relaxation by $20 \%$, whereas at low surface coverage, polymer enhances the rate relativeto that of the bare membranes. Hindered flow of solvent through the adsorbed polymer layer at high coverage appears to explain the retardation. The faster dynamics at low polymer coverage may be due to stiffening of the membrane or lateral diffusion of the dilute adsorbed polymer chains.
\end{abstract}

\section{Introduction}

Polymers confined in lyotropic lamellar phases have received theoreticall1,2 and experimental ${ }^{3-7}$ attention. Polymers change intermembrane (e.g., osmotic compressibility and phase separation) and intramembrane interactions (e.g., membrane flexibility and the mean curvature). Accordingly, new phasebehavior is expected. ${ }^{8,9}$ The effect of polymers on the bilayer properties depends on both the molecular structure of the polymer and the polymer concentration. Ther efore, it is possible to tuneor to stabilize a particular state of surfactant organization by manipulating the polymer structureand concentration.

We would expect that the polymer would affect membrane dynamics by (1) increasing bilayer mean bending modulus $\kappa$, which would speed up the relaxation, (2)

\footnotetext{
* To whom correspondence should be addressed.

† Princeton University.

‡ Argonne National Lab.

§ Institut für F estkörperforschung- Forschungszentrum J ülich.

"Rutgers University.

(1) de Gennes, P. G. J . Phys. Chem. 1990, 94, 8407

(2) Brooks, J. T.; Cates, M. E. J . Chem. Phys. 1993, 799, 5467.

(3) Brooks, J. T.; Marques, C. M.; Cates, M. E. J. Phys. II 1991, 6, 673.

(4) Dexter, D. L. J . Chem. Phys. 1953, 21, 836

(5) Iliopoulos, I.; Olsson, U. J. Phys. Chem. 1994, 98, 1500.

(6) Ligoure, C.; Bouglet, G.; Porte, G. Phys. Rev. Lett. 1993, 71, 3600.

(7) Singh, M.; Ober, R.; Kleman, M. J . Phys. Chem. 1993, 97, 11108.

(8) Yang, Y.; Prud'homme, R. K.; Richetti, P.; Marques, C. In Supramol ecular structure in confined geometries; Manne, S., Warr, G. G., Eds.; ACS Symposium Series 736; American Chemical Society: Washington, DC, 1999.

(9) Yang, B.-S.; Lal, J .; Marques, C. M.; Richetti, P.; Russel, W. B.;
} Prud'homme, R. K. Langmuir 2001, 17 (19), 5834. increasing the repulsive bilayer interaction, which would speed up therelaxation, and (3) enhancing the dissipation in the adsorbed polymer brush layer, which would slow down the relaxation. Theories regarding the effect of polymer on the bending modulus $\kappa$ are contradictory. Hiergeist and Lipowsky"10 suggested that polymers "anchored" to the membrane would increase $\kappa$. In contrast, Brooks et al. claimed that polymer "adsorption" to the membranewould decrease $\kappa .{ }^{3}$ Zilman and Granek ${ }^{11}$ found that the relaxation rate of the surfactant bilayer is inversely proportionality to $\kappa^{1 / 2}$, unlike the prediction of hydrodynamic theories which indeed predict the direct proportionality of the relaxation to $\kappa$. A possiblesoftening of the membrane due to adsorbing polymer may be considered. Hence, the observation of an increased relaxation rate of the membranein the presence of adsorbed polymers, compared to that of the bare membrane, would confirm the result of Zilman and Granek. However, we haveseen that the Zilman-Granek approximation works relatively well only for $\kappa \gg 1 \mathrm{k}_{\mathrm{B}} \mathrm{T}$. Someresearchers relate $\kappa$ to the degree of homogeneity of the spontaneous curvature, that is, the polymer density on the surface. ${ }^{12,13}$ Our previousstudy on bicontinuous microemulsion phases with amphiphilic block copolymers anchored to the surfactant monolayer has shown that themost significant contribution on the local (high $\vec{q}$ ) dynamics, observed by neutron spin-echo, comes from thefriction (viscosity) and the surfactant concentration (membrane area per unit

(10) Hiergeist, C.; Lipowsky, R. J . Phys. II 1996, 6 (10), 1465.

(11) Zilman, A. G.; Granek, R. Phys. Rev. Lett. 1996, 77, 4788

(12) Leibler, S.; Andelman, D. J . Phys. (Paris) 1987, 48 (11), 2013.

(13) Helfrich, W.; Kozlov, M. M. J . Phys. II 1994, 4 (8), 1427. 


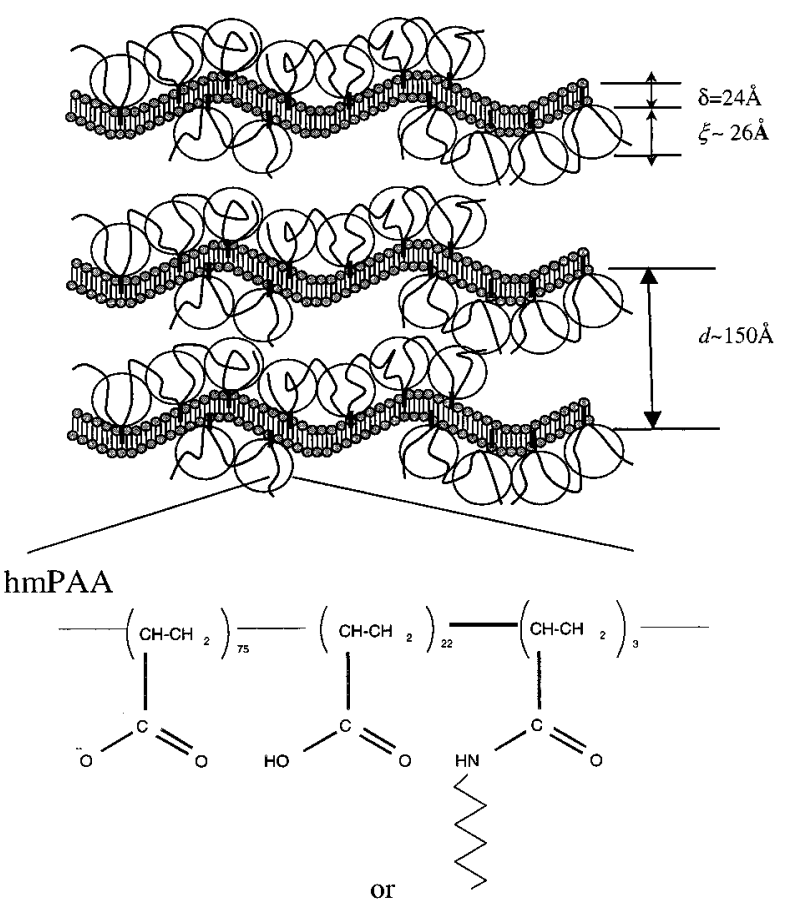

hmPEG<smiles>CNC(=O)C1NC2CCCC1NC2OCCOC1CCCC1</smiles>

Figure 1. Sketch of the anticipated structure of the mixture of hydrophobically modified polymer and surfactant lamellar phase. Thelength scales are for thesystem of membranevolume fraction $\phi=0.3$ doped with hmPAA with 3\% hydrophobe substitution. The enlarged pictures are the chemical composition of hmPAA and hmPEG.

vol ume), and no polymer effect is observed in this $\vec{q}$ range. An effect of the anchored polymer density is only observed in the hydrodynamic, low $\overrightarrow{\mathrm{q}}$, region via dynamic light scattering (DLS) measurements. There a speeding up of the effective diffusion with the polymer density (at constant structure size) suggests increased repulsive interaction between layers. ${ }^{14}$

We have previously characterized the changes induced by hydrophobically modified polymers (hm-polymers) on the static elastic properties (bilayer mean bending modulus $\kappa$ and layer compression modulus $\bar{B}$ ) of a nonionic membrane system using small-angle neutron scattering (SANS).9,15 Twohm-pol ymers havebeen investigated: (a) hydrophobically modified poly(acrylate) (hmPAA) with tetradecyl $\left(\mathrm{C}_{14}\right)$ sidegroups randomly grafted to the poly(acrylate) backbone and (b) poly(PEG $\mathrm{Pk}_{6 \mathrm{l}}$-lysine-stearylamide) (hmPEG) with equally spaced hydrophobes. ${ }^{9,15-18}$ Figure 1 sketches the structure of the interacting hydrophobically modified polymer and surfactant lamellar bilayer. The lamellar phase conformation has been confirmed by our previous SANS study.9,15 The hm-

(14) Mihailescu, M. et al. To be published.

(15) Yang, B.-S.; Lal, J .; Kohn, J .; Huang, J. S.; Russel, W. B.; Prud'homme, R. K. Langmuir 2001, i'7 (21), 6692.

(16) Yang, B.-S. Ph.D. Thesis, Princeton University, Princeton, NJ , in preparation.

(17) Heitz, C.; Pendharker, S.; Kohn, J .; Prud'homme, R. K. Macromol ecules 1999, 32, 6652.

(18) Heitz, C.; Prud'homme, R. K.; Kohn, J . Macromolecules 1999, 32,6658 . polymers are associated with the two-dimensional membrane through their hydrophobic anchoring sites. The system can be thought of as the two-dimensional surfactant membrane doped with the strings of blobs formed by the hydrophili c segments ("I oops") between hydrophobes. The interlamellar spacing $d$ is obtained from the Bragg peak position and structure factor of the scattering spectrum. The membrane thickness $\delta$ is calculated from the form factor of the scattering spectrum. The size of a blob is determined by the molecular weight of the loop. Our previous studies found that the "rigidity" (theproduct of $\kappa$ and $\bar{B}$ ) and layer compression modulus $\bar{B}$ of the membranes are independent of hydrophobe length, molecular weight, and pol ydispersity of thehm-polymers but increase with polymer concentration and hydrophobe substitution level.9,15,19 Nevertheless, the dynamics and exact value of bending modulus $\kappa$ of surfactant bilayers, with and without polymer doping, cannot be measured through the static scattering. In our previous studies, we observed contradictory results when membrane rigidity was cal culated from SANS data using the peak divergence analysi $\mathrm{s}^{20,21}$ and the excess area method. ${ }^{22}$ Measuring the membrane dynamics directly should help clarify this discrepancy.

The aim of this paper is to systematically study the effect of membrane volume fraction and addition of hmpolymers on the dynamic behavior of thesurfactant bilayer using SANS and neutron spin-echo (NSE ) spectroscopy, respectively. The so-cal led excess area method of Roux et al. ${ }^{22}$ is used to extract the bending modulus $\kappa$ from SANS data. The dynamicstructurefactors of a surfactant bilayer, with or without hm-pol ymer doping, aregiven by the NSE spectroscopy. Thestaticand dynamic measurements were compared for both bare membrane and polymer-doped membranesystems. Wehad anticipated a correspondence between increased membrane stiffness (from SANS) and increased relaxation rates of thermal fluctuations (from NSE). However, the response is more complicated. The limitations of models relating measured SANS and NSE quantitiestomembrane properties are discussed. Thefirst section of this paper describes the materials and the experimental techniques applied in this study. Thesecond section describes thestatic and dynamic data on bareand polymer-doped membranes. Thefinal section summarizes the highlights of this study.

\section{Experiments}

Systems and Materials. The surfactant lamellar phase consists of the nonionic surfactant penta(ethyleneglycol) dodecyl ether $\left(\mathrm{C}_{12} \mathrm{E}_{5} ;>99 \%\right.$, Nikko Chemical Co. Ltd., Tokyo) and 1-hexanol (>99\%, Fluka), used as received. Pure $\mathrm{C}_{12} \mathrm{E}_{5} /$ water mixtures have a wide $L_{\alpha}$ phase region at $60{ }^{\circ} \mathrm{C}$, spanning a membrane vol ume fraction $\phi$ from as low as 0.005 to order unity. ${ }^{23}$ Adding hexanol to the membrane reduces the rigidity ${ }^{24}$ and extends the wide lamellar range of the phase diagram to room temperature. The $\mathrm{C}_{12} \mathrm{E}_{5} /$ hexanol molar ratioin all of our samples is fixed at $1: 1.43$, which reduces the membrane rigidity to $k_{B} T .24$ The solvent phase is $0.1 \mathrm{M} \mathrm{NaCl}_{\left(\mathrm{D}_{2} \mathrm{O}\right)}$, which effectively screens the el ectrostatic interactions of the added anionic polymers; the Debye length $I_{D}$ is $10 \AA$ for the $\mathrm{NaCl}$ solution at $0.1 \mathrm{M}$. In the

(19) Yang, Y.; Prud'homme, R. K.; McGrath, K. M.; Richetti, P.; Marques, C. M. Phys. Rev. Lett. 1998, 80 (12), 2729.

(20) Caillè, A. H. C. R. Acad. Sci. B 1972, 274, 1733.

(21) Nallet, F.; Laversanne, R.; Roux, D. J . Phys. II France 1993, 3, 487.

(22) Roux, D.; Nallet, F.; Freyssingeas, E.; Porte, G.; Bassereau, P.; Skouri, M.; Marginan, J . Europhys. Lett. 1992, 17, 575.

(23) Strey, R.; Schomacker, R.; Roux, D.; Nallet, F.; Olsson, U. J. Chem. Soc., Faraday Trans. 1990, 86 (12), 2253.

(24) Freyssingeas, F .; Nallet, E.; Roux, D. Langmuir 1996, 12, 6028. 
Table 1. Summary of Parameters for Bare Membrane and Polymer Doped Membrane Systems Studied.

\begin{tabular}{|c|c|c|c|c|c|c|}
\hline & & & \multicolumn{4}{|c|}{ polymer-doped membrane } \\
\hline & \multicolumn{2}{|c|}{ bare membrane } & \multicolumn{2}{|c|}{ hmPAA } & \multicolumn{2}{|c|}{ hmPEG } \\
\hline & $\phi=0.2$ & $\phi=0.3$ & $\begin{array}{c}\phi=0.2 \\
\mathrm{C}_{\mathrm{p}}=2 \mathrm{wt} \%\end{array}$ & $\begin{array}{c}\phi=0.3 \\
C_{p}=2 \text { wt \% }\end{array}$ & $\begin{array}{c}\phi=0.3 \\
\mathrm{C}_{p}=0.5 \text { wt } \%\end{array}$ & $\begin{array}{c}\phi=0.3 \\
C_{p}=2 \text { wt \% }\end{array}$ \\
\hline $\begin{array}{l}\mathrm{N}_{\mathrm{b}} \\
\xi(\AA)\end{array}$ & & & & & & \\
\hline$d(\AA)$ & 144 & 99 & 154 & 103 & 103 & 104 \\
\hline$\delta(\AA)$ & 24 & 24 & 24 & 24 & 24 & 24 \\
\hline$\sigma$ & 0 & 0 & 0.82 & 0.54 & 0.07 & 0.30 \\
\hline
\end{tabular}

brine solution, the hmPAA can be viewed as an almost neutral chain with an average number of 3500 (for $M_{w}=250 \mathrm{~kg} / \mathrm{mol}$ ) backbonemonomers. When the el ectrostaticrepulsion is screened with brine, the undulation force is the dominant long-range repulsion. HmPEG is nonionic.

$\mathrm{H}$ mPAA is synthesized by thegraft reaction of precursor poly(acrylic acid) with $n$-tetradecylamine $\left(\mathrm{C}_{14}\right)$ in the presence of 1,3dicycl ohexylcarbodiimide (DCC), using n-methylpyrrolidinone (NMP) as the solvent. ${ }^{25}$ The precursor PAA (Aldrich; $\mathrm{M}_{w}=250$ $\mathrm{kg} / \mathrm{mol}, \mathrm{M}_{\mathrm{w}} / \mathrm{M}_{\mathrm{n}} \sim 2$ ) in a $35 \mathrm{wt} \%$ aqueous solution was freeze dried before use. Modified polymers were precipitated and recovered from the solution by adding concentrated $\mathrm{NaOH}(40$ wt \%) aqueous solution. The modified polymers have the same polymerization degree as the precursor with a typical structure illustrated in the enlarged picture in Figure 1. The degree of neutralization of the hmPAAs is $75 \pm 1.5 \mathrm{~mol} \%$, determined by elemental analysis, and the hydrophobe substitution level is 3 mol \%, determined by ${ }^{1} \mathrm{H}$ NMR. The distribution of the hydrophobic side chains $\left(C_{14}\right)$ along the PAA backbone is random. ${ }^{26}$

The details of synthesis and characterization of hmPEG are reported elsewhere. ${ }^{17}$ This involves synthesis of (a) Iysine stearylamide dihydrochloride, (b) bis(succinimidyloxycarbonyl PEG) (BSC-PEG), 27,28 and (c) poly(PEG $6 \mathrm{~K}_{\mathrm{k}}$-lysine-stearylamide), sequentially. Lysine stearylamide dihydrochl oride was polymerized interfacially with BSC-PEG in the presence of potassium carbonate according to a published procedure. ${ }^{27}$ The structure of the synthesized polymers was characterized by ${ }^{1} \mathrm{H}$ NMR. Molecular weight is determined by GPC and calculated relative topoly(ethyleneoxide) standards. Theweight-averaged mol ecular weight of PEG spacers $\left(m_{b}\right)$ is $6 \mathrm{~kg} / \mathrm{mol}$, and the hydrophobe is $\mathrm{C}_{18} \mathrm{H}_{37}$. The total molecular weights $\mathrm{M}_{\mathrm{w}}$ and $\mathrm{M}_{\mathrm{n}}$ of hmPEG are 112 and $78 \mathrm{~kg} / \mathrm{mol}$, respectively. That corresponds to average of 13 hydrophilic loops per polymer chain, calculated by $M_{n} / m_{b}$.

The interlamellar spacing $d$ and membrane thickness $\delta$ were determined by small-angle neutron scattering. To calculate the surface coverage of hm-pol ymers on the surfactant membrane, we regard the anchored $\mathrm{hm}$-polymer as a swollen planar coil of blobs composed of $\mathrm{N}_{\mathrm{b}}$ loops with average bl ob size $\xi$. The details for the calculation of $\mathrm{N}_{\mathrm{b}}$ and $\xi$ were reported el sewhere. ${ }^{9,15}$ The total membrane area (per unit volume) is

$$
\mathrm{A}_{\text {membrane }} \approx \frac{2}{\mathrm{~d}} \approx \frac{2 \phi}{\delta}
$$

The blobs on the surfactant membranes cover an area (per unit volume) of

$$
\mathrm{A}_{\text {polymer }} \approx \frac{\text { no. of chains }}{\text { volume }} \frac{\text { blobs }}{\text { chain }} \frac{\text { area }}{\text { blob }} \approx\left(\frac{\mathrm{C}_{\mathrm{p}} \mathrm{N}_{\mathrm{A}}}{\mathrm{M}_{\mathrm{n}}}\right) \mathrm{N}_{\mathrm{b}} \xi^{2}
$$

where $C_{p}$ is polymer concentration and $N_{A}$ is Avogadro's number. $9,15,19$ The surface coverage of polymer on the membrane is calculated by

$$
\sigma=\frac{\mathrm{A}_{\text {polymer }}}{\mathrm{A}_{\text {membrane }}}
$$

Table 1 summarizes the geometry and surface coverage of the systems applied in this study.
Sample Preparation. Thesamples were prepared by mixing surfactant $\left(\mathrm{C}_{12} \mathrm{E}_{5}\right)$, alcohol $\left(\mathrm{C}_{6} \mathrm{OH}\right)$, and stock solutions of the hm-polymers by weight in $0.1 \mathrm{M} \mathrm{NaCl}_{\left(\mathrm{D}_{2} \mathrm{O}\right)}$. In the following, we definethe "membranevolumefraction" $\phi$ as theratio of thevolume of the $\mathrm{C}_{12} \mathrm{E}_{5}$ plus $\mathrm{C}_{6} \mathrm{OH}$ divided by the total sample volume. For conversion from weight to volume fractions, the following densities $\left(\mathrm{g} / \mathrm{cm}^{3}\right)$ have been used: $0.996\left(\mathrm{C}_{12} \mathrm{E}_{5}\right), 0.820\left(\mathrm{C}_{6} \mathrm{OH}\right)$, $1.105\left(\mathrm{D}_{2} \mathrm{O}\right)$, and $0.998\left(\mathrm{H}_{2} \mathrm{O}\right)$, neglecting the effect of the added polymers on the solvent density.

Small-Angle Neutron Scattering. Thesmall-angleneutron scattering experiments were performed at Argonne National Laboratory at Argonne, IL, with the time-of-flight small-angle diffractometer (SAD). Thescattering vector $(\overrightarrow{\mathrm{q}})$ varied from 0.005 to $0.35 \AA^{-1}$. Samples were held in $1 \mathrm{~mm}$ path length quartz cells. The data were processed according to the standard procedures, including the subtraction of scattering from the solvent as background and empty cell contributions. ${ }^{9}$ Particular attention has been paid to maintaining the lamellar samples in a polycrystalline state to obtain a powder-average. Prior to every neutron run, samples were quenched to produce small liquid crystal domain size by first raising the temperature into the isotropic sponge phase range and then quickly immersing in a water bath at $\mathrm{T}=25^{\circ} \mathrm{C}$. I sotropic two-dimensional spectra are then obtained, and duplicateruns indicated no sensitivity of the d-spacing to quenching details.

Neutron Spin-Echo. TheNSE experiments wereperformed at Institut für Festkörperforschung-Forschungszentrum in J ülich, Germany. Theneutron spin-echospectrometer is a highresolution instrument suited for the study of relaxation-type motion induced by thermally excited density fluctuations in the system. It may also be viewed as an augmentation of smallanglescattering with inelastic, dynamical information. TheJ ülich NSE spectrometer uses cold neutrons issued from the $\mathrm{H}_{2}$ cold source in the $\mathrm{D}_{2} \mathrm{O}$-moderated, $20 \mathrm{MW}$ reactor. A Dornier vel ocity selector $10 \%$ fwhm (full width at half-maximum) in wavelength spread and a selective polarizing multilayer mirror provide pol arized neutrons with a wavelength of $8 \AA$. Two water-cooled copper solenoids with a maximum field integral of $0.5 \mathrm{~T} \mathrm{~m}$ (Tesla times meter) produce the precession fields for the neutrons. A cobalt-titanium supermirror assembly performs the final polarization analysis. A larger area converging collimator in front of the analyzer is used to reduce the background. The neutrons are detected by a Helium-3 array detector made of $32 \times 32$ cells with a total area of $1 \mathrm{~cm}^{2}$. The details of the NSE principles and instrument were described in detail el sewhere. ${ }^{29,30}$

The individual neutron-spin precessing in a magnetic field before and after scattering is used as timekeeper to measure the vel ocity change of the neutron during the scattering. This allows for a broad incoming neutron velocity band (10-20\%) as usual in SANS, yielding reasonable intensity simultaneously with sensitivity to relative velocity changes in the $10^{-4}-10^{-6}$ range. The spin-echo method directly measures the normalized intermediate dynamic structure factor $\mathrm{S}(\mathrm{q}, \mathrm{t}) / \mathrm{S}(\mathrm{q}, 0)$, which is proportional to the cosine Fourier transform of the scattering

(26) Magny, B.; Lafuma, F.; Iliopoulos, I. Polymer 1992, 33, 3151.

(27) Tanaka, F.; Edwards, S. F.J . N on-N ewtonian Fluid Mech. 1992 43,247

(28) J ones, M. J . Colloid I nterface Sci. 1967, 23, 36.

(29) Neutron Spin-Echo Proc. Lecture Notes in Physics; Mezei, F .; Ed.; Springer: Berlin, 1979; Vol. 128

(30) Monkenbusch, M.; Schaetzler, R.; Richter, D. Nucl. Instrum. Methods Phys. Res. 1997, A399, 301. 
(a)
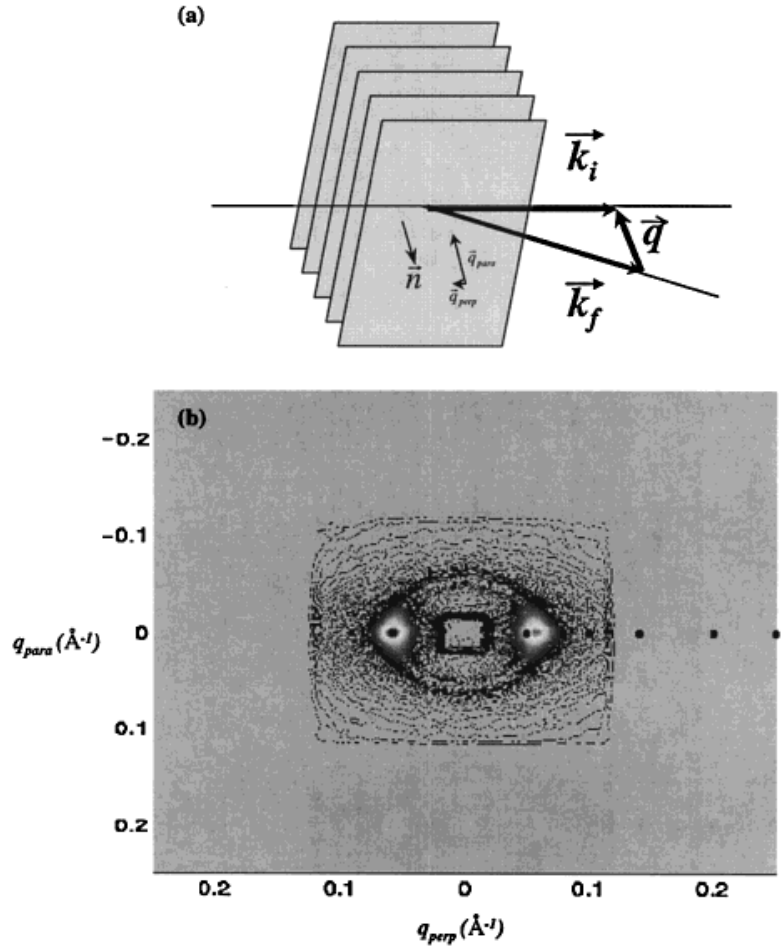

Figure 2. (a) Orientation of the ordered lamellae, incident neutron beam $\vec{k}_{i}$, scattered beam $\vec{k}_{f i}$, and scattering vector $\vec{q}$. (b) Two-dimensional SANS intensity map from an oriented lamellar sample ( $\phi=0.3$ with $2 \mathrm{wt} \% \mathrm{hmPAA})$ taken at the small-angle neutron scattering instrument (KWS-2) at IFF, J ülich, Germany. Note that $\vec{q}_{\text {para }}$ is the component of $\vec{q}$ parallel to the normal $\mathrm{n}$ (often called $\overrightarrow{\mathrm{q}}_{\mathrm{z}}$ ); $\overrightarrow{\mathrm{q}}_{\text {perp }}$ is the component perpendicular to the normal, i.e. parallel to the membrane.

function $\mathbf{S}(q, \omega)$. The information on the investigated system is gained through thescattering length density correlator $\langle\delta \rho(\vec{r}, t) \delta \rho$ $\left.\left(\vec{r}^{\prime}, 0\right)\right\rangle$ by

$\mathrm{S}(\overrightarrow{\mathrm{q}}, \mathrm{t}) \sim \iint_{\mathrm{r}, \mathrm{r}^{\prime}}\left\langle\delta \rho\left(\vec{r}^{\prime}, \mathrm{t}\right) \delta \rho\left(\vec{r}^{\prime}, 0\right)\right\rangle \exp \left[i \overrightarrow{\mathrm{q}}\left(\vec{r}-\vec{r}^{\prime}\right)\right] \mathrm{d}^{3} \vec{r} \mathrm{~d}^{3} \vec{r}^{\prime}$

where $\delta \rho(\vec{r}, \mathrm{t})$ describes the density fluctuations, at position $\vec{r}$ and time $t$, from the average scattering length density $\rho(\vec{r}, t) .{ }^{29,30}$

The lamellar phase samples were oriented by injecting the mixtures into a specially designed sample cell, which consists of a stack of very thin lamellar quartz plates $(0.2 \times 5 \times 45 \mathrm{~mm})$ in a stainless steel frame (F igure 2a). The spacing between quartz plates is $0.1 \mathrm{~mm}$. The orientation was achieved through the proximity of the neighboring quartz plates and by shear during injection. All the experiments were performed at $\mathrm{T}=25^{\circ} \mathrm{C}$. The quality of the orientation was checked by a preliminary measurement with the SANS instrument (KWS-2). The dynamic experiments were performed immediately after on the NSE spectrometer. The orientation of the lamellar stack relative to the scattering plane was chosen so that the momentum transfer vector $\vec{q}$ is perpendicular to thelamellar membrane ( $F$ igure 2a). In Figure 2b, thetwo-dimensional SANS intensity map is shown for the sample of $\phi=0.2$ doped with 2 wt $\%$ hmPAA. F rom the two-dimensional SANS intensity map (Figure 2b), adequate intensities for NSE were obtained for $\vec{q}<0.2 \AA^{-1}$. NSE experiments have therefore been performed for $\vec{q}=0.05,0.08$, $0.1,0.14,0.2$, and $0.24 \AA^{-1}$ to cover dynamics from just bel ow the Bragg peak to well beyond it.

Instrumental resolution effects were corrected by the division

$$
\frac{\mathrm{S}(\overrightarrow{\mathrm{q}}, \mathrm{t}) / \mathrm{S}(\overrightarrow{\mathrm{q}}, 0)_{\text {sample }}}{\mathrm{S}(\overrightarrow{\mathrm{q}}, \mathrm{t}) / \mathrm{S}(\overrightarrow{\mathrm{q}}, 0)_{\text {reference }}}
$$

where a sample hol der (without quartz plates) filled with carbon powder served as a reference. Duetothehigh scattering intensity of the sample, background subtraction was not necessary and not performed. The evaluation of $\mathrm{S}(\overrightarrow{\mathrm{q}}, \mathrm{t}) / \mathrm{S}(\overrightarrow{\mathrm{q}}, 0)$ was performed for each $4 \times 4$ cell group of the detector, individually. The results werethen summed into several $\vec{q}$ groups (rings) for each scattering arm angle corresponding to the $\vec{q}$ values mentioned above.

\section{Results and Discussion}

Bare Membrane. To interpret the dynamics of the membranesystems Zilman et al . consi dered an ensemble of membrane plaquettes at random orientations and calculated theeffect from the undulations bending energy and standard hydrodynamic dispersion law. ${ }^{11}$ They predict a stretched exponential relaxation in the form of

$$
S(\vec{q}, t) \cong S(\vec{q}) e^{-\left(\Gamma_{q} t\right)^{2 / 3}}
$$

where $S(q)$ is the static structure factor and $\Gamma_{q}$ is the relaxation rate.

Figure 3 shows thenormalized dynamic structurefactor $\mathrm{S}(\mathrm{q}, \mathrm{t}) / \mathrm{S}(\mathrm{q}, 0)$ for the bare membrane samples $(\phi=0.2$ and $\phi=0.3$ ). Both of our bare membrane systems exhibit a relaxation behavior that cannot be fitted by a single exponential but is well fitted by this stretched exponential. Results based on eq 6 are shown as dashed lines $(\phi=0.2)$ and full lines $(\phi=0.3)$. The $\phi=0.2$ sample relaxes faster than $\phi=0.3$ for the whole $q\left(=0.05 \AA^{-1}\right)$. The length scale I in thesystem probed by scattering vector $\vec{q}$ of NSE follows $q \sim 2 \pi / l$. The dynamics of the interacting membranes is probed at $\mathrm{q} \sim 0.04$ and $0.06 \AA^{-1}$ for $\phi=0.2(\mathrm{~d} \sim 150 \AA)$ and $\phi=0.3(d \sim 100 \AA)$, respectively. Thelocal fluctuations of the single bilayer (membrane thickness $\delta \sim 24 \AA$ ) are probed at $q>0.1 \AA^{-1}$, and in this range of $\vec{q}$ the difference in relaxation behavior becomes larger. This means that the dynamics of the single bilayer at different membrane volume fractions ( $\phi=0.2$ and $\phi=0.3$ ) are different (by about 50\%); membrane interactions are significant. This will be discussed in more detail below.

Polymer-Doped Membrane. The dynamics of the polymer-doped membranesystems wereal soinvestigated by neutron spin-echospectroscopy with the same $\vec{q}$ range and orientation as those of the bare membrane experiments. Figure 4 shows the dynamic structurefactor $\mathrm{S}(\mathrm{q}, \mathrm{t}) /$ $\mathrm{S}(\mathrm{q}, 0)$ for the samples with and without hmPAA polymer for $\phi=0.2$. The polymer-doped system also exhibits a stretched exponential relaxation behavior, similar tothat observed for the bare membrane systems. Fitted results based on eq 6 are shown as dashed lines (bare membrane) and full lines (polymer-doped membrane). At $\phi=0.2$, the polymer-doped membrane relaxes more slowly than the bare membrane for the full range of $\vec{q}$ investigated. The difference becomes much more promi nent for $q>0.1 \AA^{-1}$ where the length scales are comparable to the single bilayer thickness of $24 \AA$. For the system with the same amount of hmPAA polymer $\left(C_{p}=2\right.$ wt \%) but with more surfactant membrane area available ( $\phi=0.3$ vs 0.2 ), the addition of hmPAA also slows down the membrane relaxation but the effect is less significant (Figure 5), than at the higher surface coverage (Figure 4).

The role of surface coverage over the range of $\sigma=0.07-$ 0.30 (Table 1) was studied for the hmPE G-doped membrane systems at $\phi=0.3$ with two different polymer concentrations of 0.5 wt \% (Figure 6a) and 2 wt \% (F igure 6b). In both figures, the relaxation of the bare membrane system $(\phi=0.3)$ at corresponding $\vec{q}$ values is shown for comparison. At the higher surface coverage of $\sigma=0.30$ (Figure 6b), the polymer-doped membrane relaxes more slowly than the bare membrane, which is consistent with what is observed in the hmPAA system for similar high surface coverages of $\sigma=0.54-0.82$ (Figure 4 and Figure 5). Figure 6a shows the relaxation of the hmPEG system 


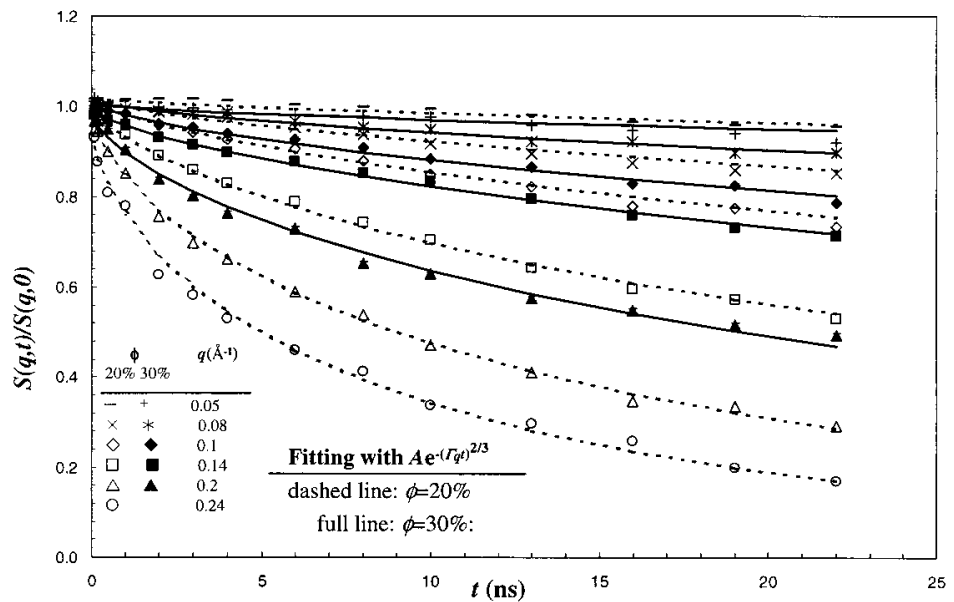

Figure 3. Normalized dynamic structure factor $\mathrm{S}(\overrightarrow{\mathrm{q}}, \mathrm{t}) / \mathrm{S}(\overrightarrow{\mathrm{q}}, 0)$ from the NSE measurement of the bare membrane systems $(\phi=0.2$ and $\phi=0.3$ ). The lines are fitting curves to eq 6 .

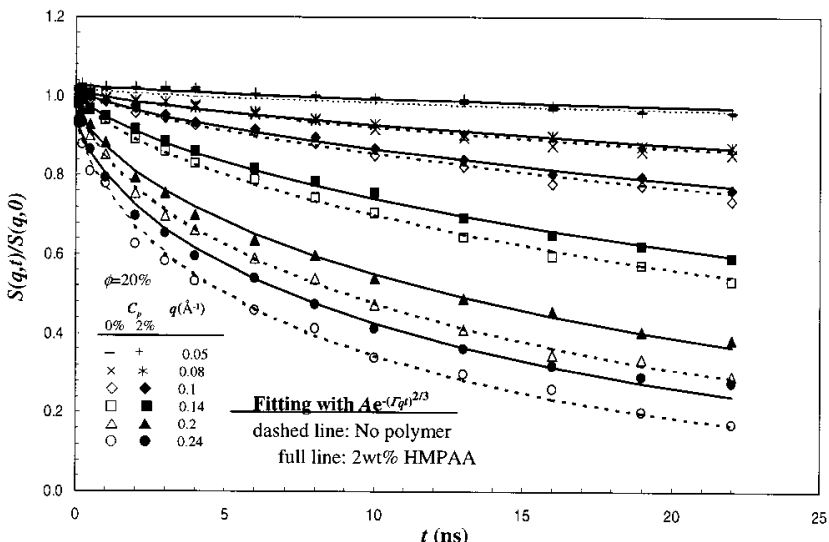

Figure 4. Normalized dynamic structure factor $S(\vec{q}, t) / S(\vec{q}, 0)$ from the NSE measurement of the bare and HMPAA-doped membrane systems at $\phi=0.2$. The lines are fitting curves to eq 6.

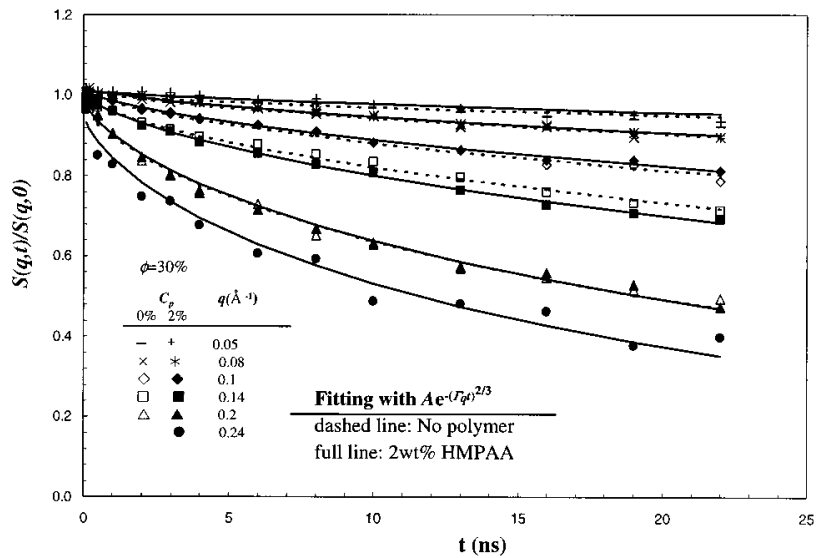

Figure 5. Normalized dynamic structure factor $S(\vec{q}, t) / S(\vec{q}, 0)$ from the NSE measurement of the bare and hmPAA-doped membrane systems $(\phi=0.3)$. Thelines are fitting curves to eq 6.

at the same membrane volume fraction $(\phi=0.3)$ at low surface coverage when the adsorbed chains should be "dilute" on the surface $(\sigma=0.07)$. At $\vec{q}>0.1 \AA^{-1}$, where $\vec{q}$ is probing the length scale comparable to the thickness of a single bilayer or the adsorbed chain, the polymerdoped membrane relaxes faster than the bare membrane.

The observation that adsorbed $\mathrm{hm}$-polymers speed up membrane relaxations at low coverage and slow down relaxations at high coverage is new. At this point, we
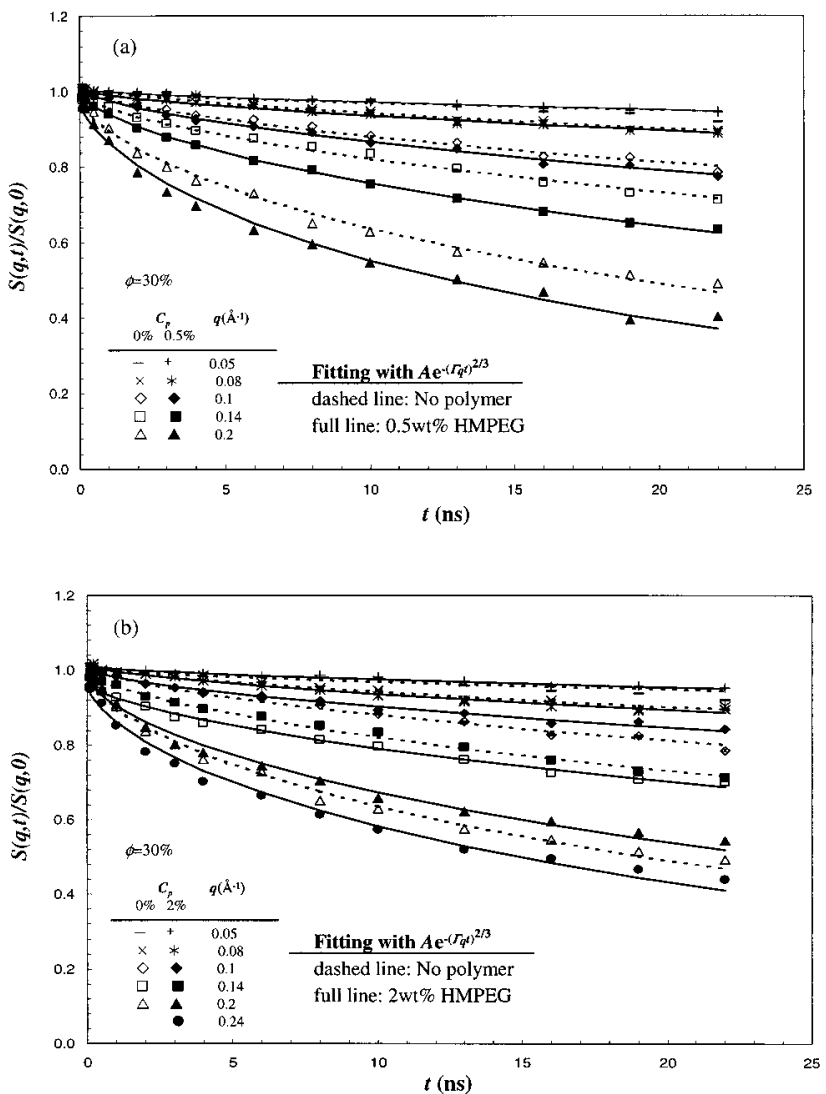

Figure 6. Normalized dynamic structure factor $\mathrm{S}(\overrightarrow{\mathrm{q}}, \mathrm{t}) / \mathrm{S}(\overrightarrow{\mathrm{q}}, 0)$ from the NSE measurement of the bare and hmPAA-doped membrane systems at $\phi=0.3$. The lines are fitting curves to eq 6. (a) $C_{p}=0.5$ wt \%; (b) $C_{p}=2$ wt \%.

propose the following physical model. There are two possibilities for the "speeding up": (1) Polymers makethe membrane stiffer, $, 1,15,31$ and ther efore the relaxations are faster (as predicted from the dispersion relation for membrane undulations, $\Gamma_{\mathrm{q}} \propto \kappa^{32,33}$ ) (Figure 7a). (2) There is a coupling between lateral polymer diffusion on the membrane surface and the relaxation of undulations in

(31) Yang, B.-S.; Lal, J .; Mihailescu, M.; Monkenbusch, M.; Richter, D.; Huang, J . S.; Russel, W. B.; Prud'homme, R. K. In LectureNotes in Physics: Neutron Spin-Echo Spectroscopy - future aspects and applications; Springer: Berlin, 2001.

(32) Messager, R.; Bassereau, P.; Porte, G.J . Phys. (Paris) 1990, 51, 1329.

(33) Brochard, F.; Lennon, J .-F. J . Phys. (Paris) 1975, 11, 1035. 
(a)

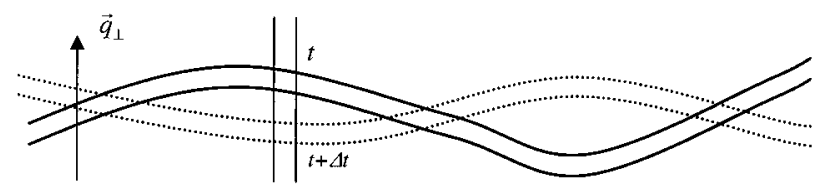

(b)
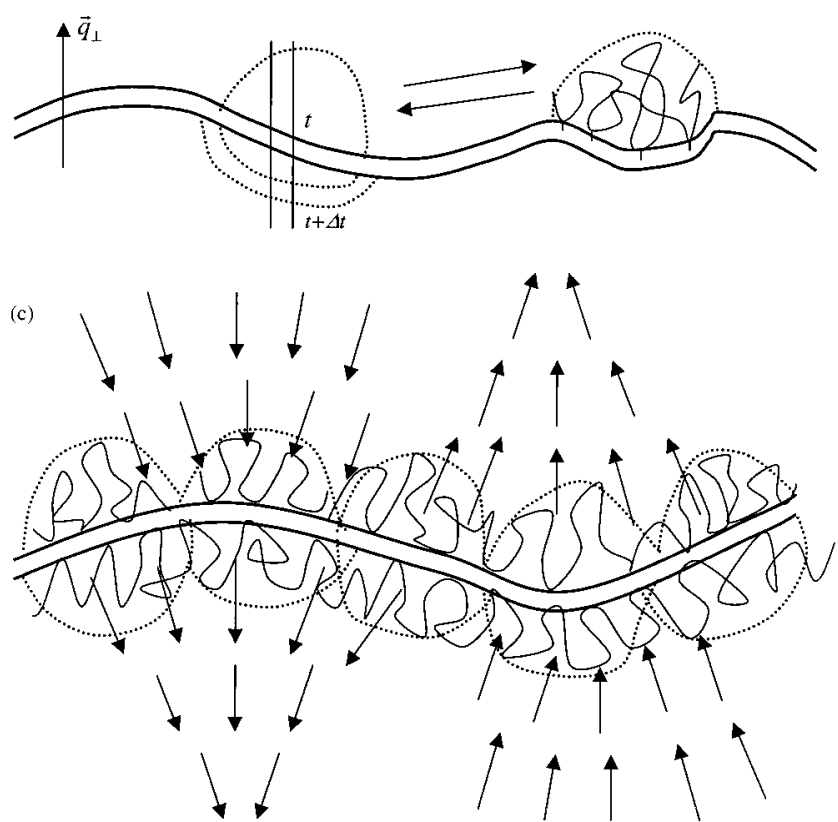

Figure 7. Sketch of the proposed polymer surface coverage effect on the membrane relaxation.

thelow surfacecoveragecase. Thepolymer diffusing across the membrane surface would "iron out" fluctuations causing a decrease in correlations (Figure 7b). This mechanism would apply only if the two-dimensional diffusion timeis comparabletothe observed $\vec{q}_{\perp}$ fluctuation time scales. The lateral diffusion coefficient of polymer (anchor) on the two-dimensional membrane is $\mathrm{D}_{\text {lateral }}=$ $10^{-7}-10^{-6} \mathrm{~cm}^{2} / \mathrm{s}^{34}$ Therefore, the relaxation time of polymer lateral diffusion $\tau_{\text {l_diff }} \sim \delta^{2} / D_{\text {lateral }} \sim 60-600 \mathrm{~ns}$, which is comparableto the membranerelaxations probed by NSE ( $\left.\tau_{\text {overall }}: 10-1500 \mathrm{~ns}\right)$.

The slowing down of the membrane relaxation at high polymer coverage may arise from increased dissipation due to flow of solvent through the polymer layer (Figure 7c). Bending the polymer brush attached to the bilayer necessitates a flux of solvent into the polymer layer in regions where it is locally stretched and out of regions where it is compressed (Figure 7c). The problem has been addressed in the context of the deflection of sol-gel layers by Scherer. ${ }^{35}$ The permeability of the polymer brush determines theresistancetoflow, and theel asticity of the bilayer with polymer determinestheelasticrestoring force. This hydrodynamic relaxation time is given by 35

$$
\tau_{\text {hyd }}=\frac{1}{2}(1-v) \frac{\eta_{\mathrm{L}} \xi^{2}}{\mathrm{DH}}
$$

where $\eta_{L}$ is the viscosity of theliquid, $v$ ( 0 for the polymer brush) is the Poisson ratio, $\mathrm{D}$ is the permeability, and $\mathrm{H}$

(34) Lenaz, G.; Samori, B.; Fato, R.; Battino, M.; Castelli, G. P.; Domini, I. Biochem. Cell Biol. 1992, 70 (6), 504.

(35) Scherer, G. W. J . Non-Cryst. Solids 1996, 204, 73.
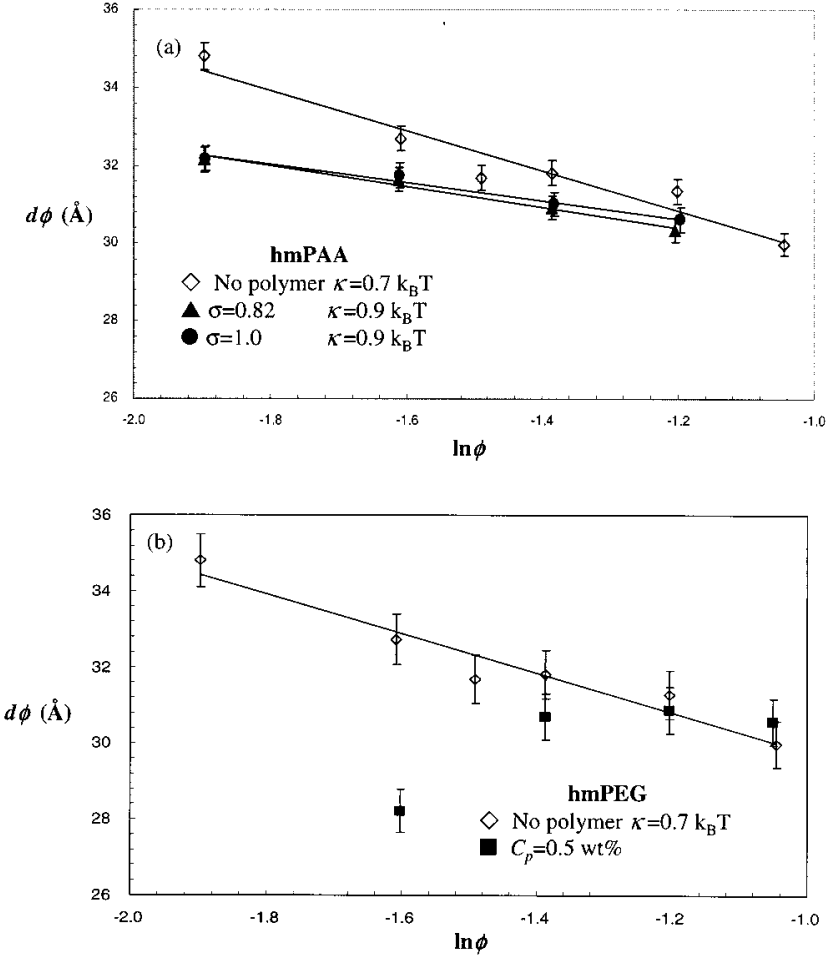

Figure 8. The measured values of $\mathrm{d} \phi$ vs $\ln \phi$ over the whole dilution rangefor (a) thehmPAA systems with different surface coverages and (b) the hmPEG system.

$=(1-v) \mathrm{E} /[(1+v)(1-2 v)]$ is the longitudinal modulus of the polymer layer, with $E$ being the Young's modulus (assumed equal to that of the surfactant membrane). The permeability of the PAA layer can be estimated by D $\eta_{\mathrm{L}} /\left(2.1 \times 10^{12} \omega^{3 / 2}\right)$, with $\omega$ being the mass fraction of polymer in the brush layer. ${ }^{36}$ The Young's modulus $\mathrm{E}$ is related to the bending modulus $\kappa$ by $\kappa=\mathrm{E} \delta^{3} / 12\left(1-v_{\mathrm{m}}{ }^{2}\right)$, where $v_{\mathrm{m}}$ is the Poisson ratio of the surfactant membrane, which is $\sim 0.5$ assuming the membrane is incompressible. ${ }^{37,38} \mathrm{~F}$ or the hmPEG-doped membrane system at $\phi=$ 0.3 and $C_{p}=2$ wt \%, the estimated hydrodynamic relaxation time $\tau_{\text {hyd }}$ for solvent toflow through the polymer layer is $\sim 900 \mathrm{~ns}$, assuming the bending modulus $\kappa \sim 1$ $\mathrm{k}_{\mathrm{B}} T, 9,15,31$ This time scale is again comparable to that of the relaxation of polymer-doped membranes, which supports the hypothesis of slowed dynamics due to viscous dissipation in the adsorbed polymer layer.

Excess Area Method. The bending modulus $\kappa$ can be extracted from SANS data using theexcess area method. ${ }^{22}$ The relationship between the interlamellar distance $d$, membrane volume fraction $\phi$, and membrane thickness $\delta$ depends on the bilayer mean bending modulus $\kappa$ according to ${ }^{19,22}$

$$
\frac{\phi \mathrm{d}}{\delta}=1+\frac{1}{4 \pi\left(\kappa / \mathrm{k}_{\mathrm{B}} \mathrm{T}\right)} \ln \left[\frac{\delta}{\phi \mathrm{b}} \sqrt{\frac{32\left(\kappa / \mathrm{k}_{\mathrm{B}} \mathrm{T}\right)}{3 \pi}}\right]
$$

where $b$ is a mi croscopic cutoff length taken to be $7 \AA .{ }^{19,24}$ The second term in theright-hand side of eq 8 represents the "excess area" associated with undulations in the membrane bilayer.

(36) Tokita, M. In Advances in Polymer Science; Springer-Verlag: Berlin, 1993; Vol. 110.

(37) Landau, L. D.; Lifschitz, E. M. Theory of Elasticity, 6th ed.; Akademie Verlag: Berlin, 1989.

(38) Simson, R.; Wallraff, E.; Faix, J .; Niewohner, J .; Gerisch, G.; Sackmann, E. Biophys. J. 1998, 74, 514. 

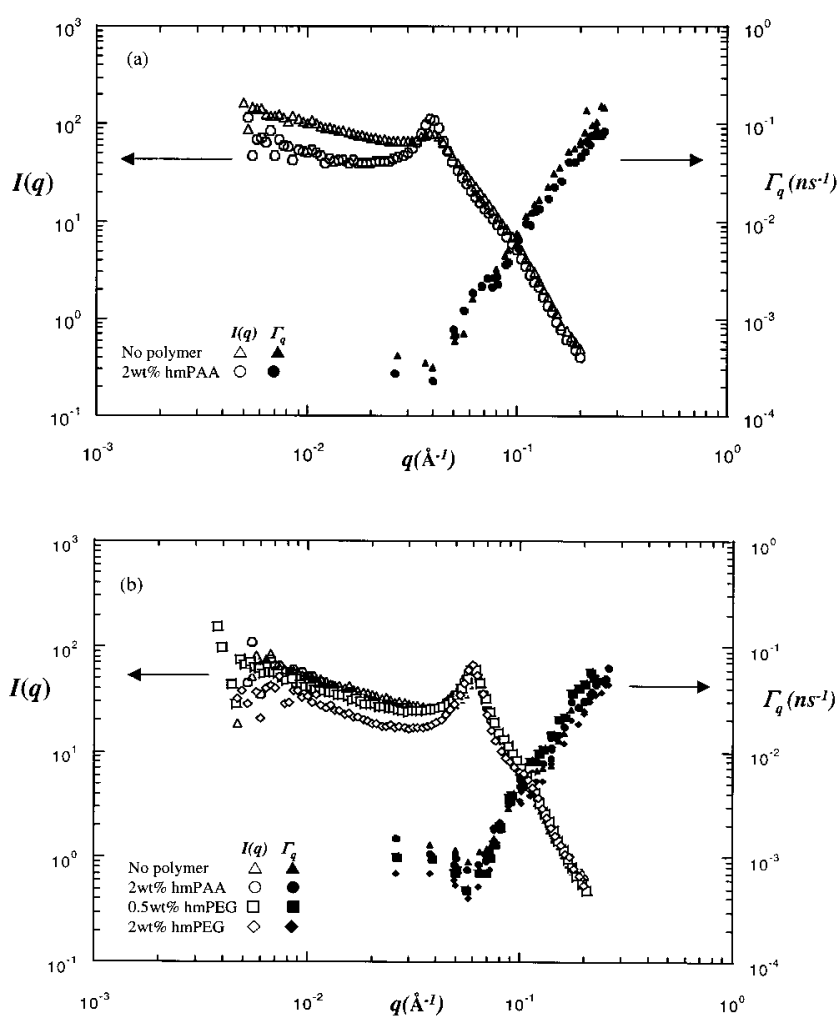

Figure 9. The $\vec{q}$ dependence of relaxation rate $\Gamma_{q}$ obtained from the fitting to eq 6 and corresponding SANS spectrum for the bare and polymer-doped membrane systems: (a) $\phi=0.2$ (b) $\phi=0.3$.

The interlamellar distance $d$ as a function of the membrane volume fraction $\phi$ was determined from the first-order Bragg peak position qo of the small-angle neutron scattering spectra; $d=2 \pi / q_{0}$ at constant hexanol/ $\mathrm{C}_{12} \mathrm{E}_{5}$ mole ratio (=1.43) and temperature $\left(25^{\circ} \mathrm{C}\right)$. For these conditions, the membrane volume fraction was varied $(0.15 \leq \phi \leq 0.35)$ within the single lamellar phase region. The best fit of the data with eq 8 gives the membrane thickness $\delta$ and the bending modulus $\kappa$. Increasing the polymer concentration at constant membrane volume fraction increases the polymer surface coverage. Figure 8a shows data for hmPAA-doped membrane samples with changing membrane volume fraction at constant surface coverage $\sigma$. Samples with two different surface coverages ( $\sigma=0.82$ and 1.0) within the homogeneous lamellar phase region yield bending constants $\kappa$ of $0.9 \pm 0.2 \mathrm{k}_{\mathrm{B}} \mathrm{T}$. However, for the hmPEG-doped membrane at low surfacecoverage $(\sigma<0.06)$ shown in Figure $8 b$, the best fit line of $\mathrm{d} \phi$ versus In $\phi$ gives a positive slope, which would be interpreted as decreasing the membrane elasticity upon the addition of polymer. Our previous study using the peak di vergence method to measure membrane stiffness showed that the hmPE G polymers increased the rigidity, that is $\kappa \bar{B}$, of the membrane phase. ${ }^{9,15}$ We believe that theincrease in area for the polymer-doped membrane at low polymer coverageis caused by a local bending of the membrane bilayer under the tension from the multiply attached polymer chain (Figure $7 \mathrm{~b}$ ). This would account for excess area that is not necessarily related to membrane flexibility or undulations. ${ }^{22}$

The basis of the excess area method depends on the bending modulus $\kappa$ of the membrane being independent of themembranevolumefraction, sothat $\kappa$ can beobtained from a dilution series at several $\phi$ values. However, the NSE results show that membrane dynamics depends on membrane separation (i.e., $\phi$ ) for bare membranes or for
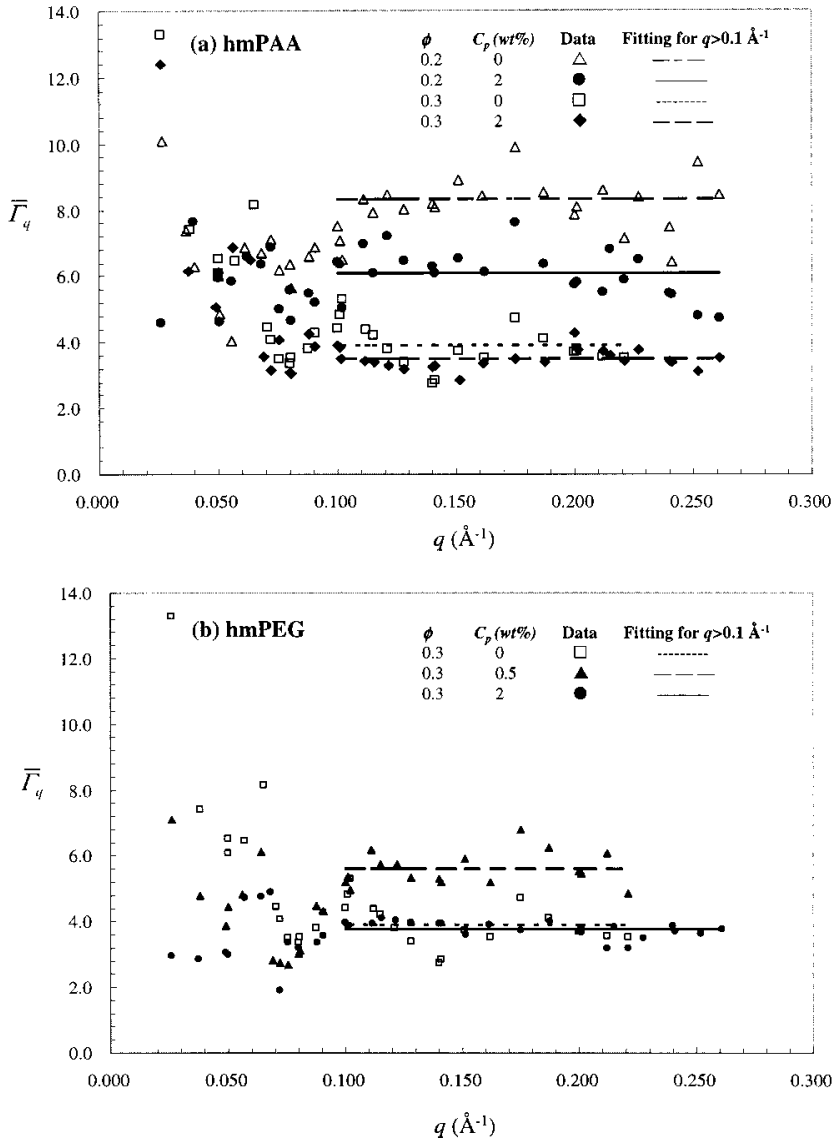

Figure 10. The $q$ dependence of the normalized relaxation rate $\bar{\Gamma}_{\mathrm{q}}=\Gamma_{\mathrm{q}} \mathrm{S}(\mathrm{q}) / \mathrm{q}^{3}$ : (a) hmPAA-doped membranes; (b) hmPEGdoped membranes.

polymer-doped membranes. The compression modulus $\bar{B}$ is significant. Therefore, for this surfactant system the elastic constant cannot be obtained from the excess area method.

Comparison between SANS and NSE Data. The relaxation rate $\Gamma_{\mathrm{q}}$ obtained from the fitting to eq 6 over the range of $\vec{q}$ from 0.03 to $0.26 \AA^{-1}$ is shown in F igure 9. Parts $a$ and $b$ of Figure 9 show the data for bare and polymer-doped membranes at $\phi=0.2$ and $\phi=0.3$, respectively. The corresponding small-angle scattering spectra are also plotted for comparison. For q at which theinterlamellar interaction is probed, thelocation of the minimum of $\Gamma_{\mathrm{q}}$ is comparable to the peak position of the static SANS spectra $\left(q_{0} \sim 0.04 \AA^{-1}\right.$ for $\phi=0.2 ; q_{0} \sim 0.06$ $\AA^{-1}$ for $\left.\phi=0.3\right)$. This observation is consistent with the behavior of $S(q) \sim 1 / \Gamma_{q}{ }^{39}$ At high $q$ values $\left(q \geq 0.1 \AA^{-1}\right)$, which reflect smaller length scales with $S(q) \approx 1, \Gamma_{q}$ scales as $\mathrm{q}^{3}$. The universal scaling rule of $\Gamma_{\mathrm{q}} \sim \mathrm{q}^{3}$ has proven accurate in both lamellar and sponge systems. ${ }^{11,32,33,40}$

Parts $a$ and $b$ of Figure 10 show the $q$ dependence of the normalized relaxation rate $\bar{\Gamma}_{\mathrm{q}}=\mathrm{S}(\mathrm{q}) \Gamma_{\mathrm{q}} / \mathrm{q}^{3}$ for the $\mathrm{hmPAA}$ and hmPEG-doped membranes and bare membranes, respectively. The normalized relaxation rate $\bar{\Gamma}_{\mathrm{q}}$ is al most independent of q beyond the Bragg peak, with average values shown in lines. However, at q in the vicinity of or below the peak the normalized relaxation rates are not distinguishable. In Figure 10a, the relaxation rate with 2 wt $\%$ ( $\sigma=0.82$, eq 3 and Table 1$)$ hmPAA is significantly slower than for the bare membrane at $\phi=0.2$ but only

(39) Farago, B.; Richter, D.; Huang, J . S.; Safran, S. A.; Milner, S. T. Phys. Rev. Lett. 1990, 65, 3348.

(40) Kawasaki, K. Ann. Phys. 1970, 61, 1. 


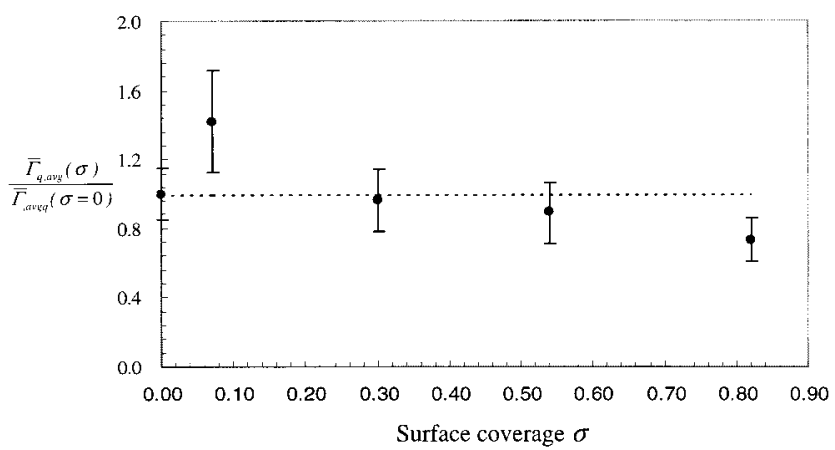

Figure 11. Dependence on the surface coverage $\sigma$ of the ratio of the average normalized relaxation rate to that of the corresponding bare membrane, for both hmPAA- and hmPEGdoped membranes.

slightly slower at $\phi=0.3(\sigma=0.54)$. Figure 10b shows that membranes at $\phi=0.3$ containing hmPAA at $\mathrm{C}_{\mathrm{p}}=2$ wt \% relax a bit slower than the bare membrane, while at $C_{p}=0.5 \mathrm{wt} \%$ polymer the relaxation is accelerated relativetothe bare membrane. I n Figure 11, weillustrate the dependence on the surface coverage $\sigma$ of the ratio of the average normalized relaxation rate $\bar{\Gamma}_{q, \text { avg }}$ above $q=$ $0.1 \AA^{-1}$ to that of the corresponding bare membrane for both hmPAA- and hmPEG-doped membranes. The re laxation rate depends subtly on the surface coverage of polymer, slowing down by $20 \%$ at high surface coverage and increasing slightly at low coverage, relative to the bare membranes.

\section{Summary}

This work is a small-angle neutron scattering and neutron spin-echo study of the static and dynamic behavior of surfactant bilayer membranes comprising $\mathrm{C}_{12} \mathrm{E}_{5}$ and hexanol. The effects of membrane volume fraction and the addition of hydrophobically modified polymers were investigated. We observed a difference in the relaxation behavior for two bare membrane systems $(\phi=0.2$ and $\phi=0.3)$. This intermembrane dependence violates the assumptions inherent in "single-membrane theories" (e.g., the excess area method and the Zilman and Granek model $\left.{ }^{11}\right)$. We are currently working on identifying the hydrodynamic modes probed by $\vec{q}$ in our oriented lamellar phase and developing a quantitative model of membranedynamics that includes interlamellar interactions and single-membrane fluctuations.

Both the bare and polymer-doped membranes exhibit the relaxation behavior that is well fitted by a stretched exponential of $\mathrm{S}(\overrightarrow{\mathrm{q}}, \mathrm{t}) \cong \mathrm{S}(\overrightarrow{\mathrm{q}}) \mathrm{e}^{-\left(\Gamma_{\mathrm{q}} \mathrm{t}\right) / 3}$. At high surface coverage, polymer doping sl ows the membranerelaxation and the effect is more prominent for large $\vec{q}$ in which the dynamics of the single bilayer is probed. A calculation of the slowing of dynamics due to limited permeability in the adsorbed polymer brush layer shows the time scales are comparable to those observed by NSE . I n contrast, polymers speed up the relaxation at l ow surface coverage. The increased relaxation rates at low polymer coverage are consistent with either stiffening of the membrane or lateral diffusion of the adsorbed polymers on the surface. The $\vec{q}$ ranges covered by the static and dynamic measurements coincide. The slowest relaxation rate occurs at length scales (i.e., $\vec{q}$ vectors) of the diffraction peak corresponding to interlamellar spacings. Fluctuations at shorter length scales (i.e., higher q values) relax at faster rates. While the theory of Zilman and Granek ${ }^{11}$ predicts the $\Gamma_{q} \propto q^{3}$ relaxation rate that we observe, the theory is a single-membrane theory that assumes dynamics to be independent of interlamellar spacing. This is contradicted by our data. A dynamictheory of multilamellar membrane stacks along the lines of the Nallet et al. theory ${ }^{21}$ for membraneaveragestatic properties is requi red. Progress al ong those lines has been made by Ning Lei; 41 however, the results are not in a tractable form at this time. The qual ity of the NSE data available invites the development of new theories of the dynamics of Iamellar phase liquid crystalline fluids.

Acknowledgment. Weacknowledgefinancial support from the Princeton Center for Complex Materials, a MRSEC funded through NSF Grant DMR 9809483. F or polymer synthesis, support was received from N I H Grant HL-60416 and the New J ersey Center of Biomaterials.

\section{LA010646K}

(41) Lei, N. Ph.D. Thesis, Rutgers U niversity, Piscataway, NJ , 1993. 